\title{
THE EULER CHARACTERISTIC OF PROJECTIVELY FLAT MANIFOLDS WITH AMENABLE FUNDAMENTAL GROUPS
}

\author{
HYUK KIM AND HYUNKOO LEE
}

(Communicated by James E. West)

\begin{abstract}
The Euler characteristic of a closed projectively flat manifold with amenable fundamental group is shown to be nonnegative, and in fact zero if we further assume that the developing map is injective and the fundamental group is infinite.
\end{abstract}

In this paper, we will show that the Euler characteristic of a closed projectively flat manifold $M$ is nonnegative if the fundamental group $\pi_{1} M$ of $M$ is amenable. If furthermore a developing map of $M$ is injective, then it is also shown that the Euler characteristic vanishes when $\pi_{1} M$ is infinite and amenable. A projectively flat manifold is a smooth manifold which has a cover of coordinate charts $\left\{U_{\alpha}, \varphi_{\alpha}\right\}$, where $\varphi_{\alpha}: U_{\alpha} \rightarrow \mathbf{R} P^{n}$ (or $\mathbf{S}^{n}$ ) is a coordinate chart for each $\alpha$ such that the coordinate transition $\varphi_{\beta} \circ \varphi_{\alpha}^{-1}$ is a restriction of a projective transformation on $\mathbf{R} P^{n}$ (or $\mathbf{S}^{n}$ ). This is a special case of a $(G, X)$-manifold when $X=\mathbf{R} P^{n}$ and $G=\operatorname{PGL}(n+1, \mathbf{R})$. (See, for instance, [NY, Th] for a general notion of $(G, X)$-manifold.)

Note that the classical space-forms of constant sectional curvature are special cases of projectively flat manifolds and its Euler characteristic in general can assume any sign by a generalized Gauss-Bonnet theorem. For example, a closed Euclidean space-form has vanishing Euler characteristic since its curvature is zero, and its fundamental group is virtually abelian by Bieberbach and hence amenable. (See [Gr] for basic facts about amenable groups.) More generally, it has long been conjectured [Mi, Go] that the Euler characteristic of a closed affinely flat manifold $M$ vanishes. (Affinely flat manifolds are $(G, X)$ manifolds with $X=\mathbf{E}^{n}=$ Euclidean $n$-space and $G=\operatorname{Aff}(n, \mathbf{R})$ and, hence, form a subclass of projectively flat manifolds.) This conjecture has been answered affirmatively when $\pi_{1} M$ is amenable [HT] and generalized to projectively flat manifolds whose developing image lies in an affine space assuming again $\pi_{1} M$ is amenable [KL]. Note that the developing image of a hyperbolic manifold lies inside the unit ball in an affine space using a projective model,

Received by the editors September 3, 1991.

1991 Mathematics Subject Classification. Primary 57R20.

Key words and phrases. Euler characteristic, projectively flat manifold, polyhedral Gauss-Bonnet formula.

Research supported by SNU-Daewoo program and GARC-KOSEF.

(C) 1993 American Mathematical Society $0002-9939 / 93 \$ 1.00+\$ .25$ per page 
but its fundamental group is not amenable. A closed spherical space-form is uniformized by the standard unit sphere, and hence its Euler characteristic is positive if the dimension is even. In fact, the only even-dimensional closed spherical spaces are spheres and real projective spaces. The proofs of the aforementioned results generalize these classical facts.

The techniques we employ in the proof are basically the same as those used in [KL] but are in the spherical setting. We use a generalized Gauss-Bonnet formula in terms of angles of simplices in a triangulation. Since we are in a non-Riemannian situation and the notion of angle is not well defined, we take advantage of amenability of a holonomy group to replace an angle by an average of the corresponding angles that appeared in the developing image.

Let $s^{n}$ be a spherical simplex lying in the standard unit sphere $\mathbf{S}^{n} \subset \mathbf{R}^{n+1}$ so that each of its $(n-1)$-dimensional faces is a part of great hyperplanes $P_{i}$, $i=1,2, \ldots, n+1$, in general position. $P_{i}$ is the intersection of $\mathbf{S}^{n}$ with an $n$-dimensional subspace of $\mathbf{R}^{n+1}$. Let $f_{i}$ be the characteristic function of the positive side of $P_{i}$ which, by definition, is the half of $\mathbf{S}^{n}$ bisected by $P_{i}$ that contains $s^{n}$.

If $s^{r}<s^{n}$ is an $r$-dimensional face of $s^{n}$ given by $s^{r}=P_{i_{1}} \cap \cdots \cap P_{i_{n-r}} \cap s^{n}$, then the angle at $s^{r}$ in $s^{n}$, denoted by $\alpha\left(s^{r}, s^{n}\right)$, is defined as

$$
\alpha\left(s^{r}, s^{n}\right)=\int_{\mathbf{S}^{n}} f_{i_{1}} \cdots f_{i_{n-r}} d \mathrm{vol},
$$

where $d$ vol is the standard measure on $\mathbf{S}^{n}$ normalized so that $\int_{\mathbf{S}^{n}} d \mathrm{vol}=1$. Clearly, $\operatorname{vol}\left(s^{n}\right)=\int_{\mathbf{S}^{n}} f_{1} \cdots f_{n+1} d$ vol and the volume of $\bar{s}^{n}$, the antipodal image of $s^{n}$, is given by $\operatorname{vol}\left(\bar{s}^{n}\right)=\int_{\mathbf{S}^{n}}\left(1-f_{1}\right) \cdots\left(1-f_{n+1}\right) d \mathrm{vol}$. Now as in [Ho, p. 29],

$$
\begin{aligned}
\operatorname{vol}\left(s^{n}\right) & =\operatorname{vol}\left(\bar{s}^{n}\right)=\int_{\mathbf{S}^{n}}\left(1-f_{1}\right) \cdots\left(1-f_{n+1}\right) d \text { vol } \\
& =\sum_{r=0}^{n} \sum_{s^{r}<s^{n}}(-1)^{n-r} \alpha\left(s^{r}, s^{n}\right)+(-1)^{n+1} \int_{\mathbf{S}^{n}} f_{1} \cdots f_{n+1} d \mathrm{vol},
\end{aligned}
$$

and hence we get a spherical Gauss-Bonnet formula

$$
\sum_{r=0}^{n} \sum_{s^{r}<s^{n}}(-1)^{r} \alpha\left(s^{r}, s^{n}\right)=\left(1+(-1)^{n}\right) \operatorname{vol}\left(s^{n}\right) .
$$

Note that when $n=2$, (2) gives the classical Gauss-Bonnet formula (rather a Harriot's formula) for a spherical triangle.

Let $k\left(s^{n}\right)$ denote the left-hand side of $(2)$ so that $k\left(s^{n}\right)=2 \operatorname{vol}\left(s^{n}\right)$ for $n$ even.

Let $M^{n}$ be a closed projectively flat manifold with a geometric triangulation $K$ consisting of triangles whose developing images are spherical simplices. (We will use $\mathbf{S}^{n}$ as a model space instead of $\mathbf{R} P^{n}$.) Such a geometric triangulation can always be found as follows : Start with any smooth triangulation on $M$ and assume each simplex lies in a geometric coordinate chart via subdivision if necessary. Then "straighten" the faces in $\mathbf{S}^{n}$; this straightening process does not depend on the choice of geometric coordinate charts since the coordinate transition maps are projective. Now for a maximal-dimensional geometric simplex $\sigma^{n}$ in $K$ and its face $\sigma^{r}<\sigma^{n}$, we associate a real number $\alpha\left(\sigma^{r}, \sigma^{n}\right)$ for 
each pair $\left(\sigma^{r}<\sigma^{n}\right)$. Let

$$
\begin{gathered}
k\left(\sigma^{n}\right)=\sum_{r=0}^{n}(-1)^{r} \sum_{\sigma^{r}<\sigma^{n}} \alpha\left(\sigma^{r}, \sigma^{n}\right), \\
S\left(\sigma^{r}\right)=\sum_{\sigma^{r}<\sigma^{n}} \alpha\left(\sigma^{r}, \sigma^{n}\right),
\end{gathered}
$$

and

$$
d(\nu)=\sum_{r=0}^{n} \frac{(-1)^{r}}{r+1} \sum_{\nu \in \sigma^{r}}\left(1-S\left(\sigma^{r}\right)\right),
$$

where $\nu$ is a vertex of $K$. Note that $d(\nu)=0$ for a vertex in a local triangulation around $\nu$ on $\mathbf{S}^{n}$ if $\alpha\left(\sigma^{r}, \sigma^{n}\right)$ is really an angle given by (1). Now it is not difficult to verify the following polyhedral Gauss-Bonnet formula for the Euler characteristic $\chi(M)$ in terms of angles:

$$
\sum_{\nu \in K} d(\nu)+\sum_{\sigma^{n} \in K} k\left(\sigma^{n}\right)=\chi(M) .
$$

The proof is just the rearrangement of terms $\alpha\left(\sigma^{r}, \sigma^{n}\right)$ which cancel out each other in the left-hand side of (3), leaving only $\sum_{r=0}^{n} \sum_{\sigma^{r} \in K}(-1)^{r}$ which, of course, equals $\chi(M)$. (See [KL] for a proof.)

We use (3) in a crucial way to prove the following theorems.

Theorem A. Let $M$ be a closed projectively flat manifold with amenable holonomy group. Then the Euler characteristic of $M$ is nonnegative. In particular, this holds if the fundamental group is amenable.

Proof. Choose any geometric triangulation $K$ on $M$, and let $(\phi, D)$ : $\left(\pi_{1} M, \widetilde{M}\right) \rightarrow\left(\operatorname{PGL}(n+1, \mathbf{R}), \mathbf{S}^{n}\right)$ be an (equivariant) developing pair, where $D: \widetilde{M} \rightarrow \mathbf{S}^{n}$ is a developing map of a universal covering $\widetilde{M}$ of $M$ into the model space $X=\mathbf{S}^{n}$ and $\phi: \pi_{1} M \rightarrow \phi\left(\pi_{1} M\right)=H \subset \operatorname{PGL}(n+1, \mathbf{R})$ is a holonomy representation. (See [NY] or [Th], for example, for the notion of a developing pair.) For each pair $\sigma^{r}<\sigma^{n}$, choose and fix, once and for all, any developing image $s_{0}^{r}<s_{0}^{n}$ of a lifting of $\sigma^{r}<\sigma^{n}$ in $\widetilde{M}$. Any other image $\left(s^{r}, s^{n}\right)$ can be written as $h\left(s_{0}^{r}, s_{0}^{n}\right)$ for some $h \in H$. Let $\alpha_{\left(\sigma^{r}, \sigma^{n}\right)}: H \rightarrow \mathbf{R}$ be a nonnegative angle function defined as $\alpha_{\left(\sigma^{r}, \sigma^{n}\right)}(h)=\alpha\left(s^{r}, s^{n}\right)$, where $\left(s^{r}, s^{n}\right)=h\left(s_{0}^{r}, s_{0}^{n}\right)$ and $\alpha\left(s^{r}, s^{n}\right)$ is the angle given in (1). Let $m$ be a right invariant mean of the amenable group $H$ and let $\alpha\left(\sigma^{r}, \sigma^{n}\right)=m\left(\alpha_{\left(\sigma^{r}, \sigma^{n}\right)}\right)$. Given $s^{n}$, and for each $s^{r}<s^{n}$, there is $h_{\left(s^{r}, s^{n}\right)} \in H$ such that $\left(s^{r}, s^{n}\right)=h_{\left(s^{r}, s^{n}\right)}\left(s_{0}^{r}, s_{0}^{n}\right)$, and hence for $n=$ even, (2) gives

$$
\begin{aligned}
0 & \leq 2 \operatorname{vol}\left(s^{n}\right)=k\left(s^{n}\right) \\
& =\sum_{r=0}^{n} \sum_{s^{r}<s^{n}}(-1)^{r} \alpha\left(s^{r}, s^{n}\right)=\sum \sum(-1)^{r} \alpha\left(h_{\left(s^{r}, s^{n}\right)}\left(s_{0}^{r}, s_{0}^{n}\right)\right) \\
& =\sum \sum(-1)^{r} \alpha_{\left(\sigma^{r}, \sigma^{n}\right)}\left(h_{\left(s^{r}, s^{n}\right)}\right)=\sum \sum(-1)^{r} h_{\left(s^{r}, s^{n}\right)} \cdot \alpha_{\left(\sigma^{r}, \sigma^{n}\right)}(e),
\end{aligned}
$$

where $e$ is the identity element in $H$ and the canonical action of $H$ on the bounded functions $f$ of $H$ is given by $h \cdot f(x)=f(x \cdot h), h, x \in H$. If we denote $k_{s^{n}}=\sum_{r=0}^{n} \sum_{s^{r}<s^{n}}(-1)^{r} h_{\left(s^{r}, s^{n}\right)} \cdot \alpha_{\left(\sigma^{r}, \sigma^{n}\right)}$ so that $k_{s^{n}}(e)=k\left(s^{n}\right)$, then one 
readily checks that $k_{s^{n}}(h)=k\left(h\left(s^{n}\right)\right)=2 \operatorname{vol}\left(h\left(s^{n}\right)\right), h \in H$, and hence obtains a positive bounded function $k_{s^{n}}: H \rightarrow \mathbf{R}$. Therefore

$$
\begin{aligned}
0 & \leq m\left(k_{s^{n}}\right)=\sum \sum(-1)^{r} m\left(h_{\left(s^{r}, s^{n}\right)} \cdot \alpha_{\left(\sigma^{r}, \sigma^{n}\right)}\right) \\
& =\sum \sum(-1)^{r} m\left(\alpha_{\left(\sigma^{r}, \sigma^{n}\right)}\right)=\sum \sum(-1)^{r} \alpha\left(\sigma^{r}, \sigma^{n}\right)=k\left(\sigma^{n}\right) .
\end{aligned}
$$

Now apply the same argument to show that $d(\nu)=0$ for each vertex $\nu \in K$. Given a developing image $v$ of a lifting of $\nu$, the (developed) angles around $v$ are again holonomy images of the reference angles $\left(s_{0}^{r}, s_{0}^{n}\right)$. We obtain a bounded function $d_{v}: H \rightarrow \mathbf{R}$ in the same way as we obtain $k_{s^{n}}$, so that

$$
d_{v}(e)=d(v)=\sum_{r=0}^{n} \frac{(-1)^{r}}{r+1} \sum_{v \in s^{r}}\left(1-S\left(s^{r}\right)\right),
$$

where

$$
S\left(s^{r}\right)=\sum_{s^{r}<s^{n}} \alpha\left(s^{r}, s^{n}\right) \quad \text { and } \quad d_{v}(h)=d(h(v)) .
$$

But this time, $S\left(s^{r}\right)=1$ holds trivially and hence $d_{v}$ is a zero function and $0=m\left(d_{v}\right)=d(\nu)$. Now the polyhedral Gauss-Bonnet formula (3) gives the desired nonnegativity of the Euler characteristic. Q.E.D.

Note that $\frac{1}{2} k\left(\sigma^{n}\right)$ can be thought of as an average volume of the developing images of $\sigma^{n}$, and thus $\frac{1}{2} k(M)=\frac{1}{2} \sum_{\sigma^{n} \in K} k\left(\sigma^{n}\right)$ is a volume of $M$ with respect to an invariant mean $m$, which of course does not depend on the choice of $m$ since it is equal to $\frac{1}{2} \chi(M)$. We can obtain more detailed information on the Euler characteristic in certain cases when we know more about developing maps. For example, if the developing image $D(F)$ of a fundamental domain $F$ in the induced triangulation of $\widetilde{M}$ is the whole $\mathbf{R} P^{n}$ (or misses a set of measure zero), then

$$
\chi(M)=k(M)=\sum_{\sigma^{n} \in K} k\left(\sigma^{n}\right)=m\left(k_{D(F)}\right),
$$

where $k_{D(F)}=\sum_{s^{n} \in D(F)} k_{s^{n}}$, and hence $\chi(M) \geq 1$ since for each $h \in H$, $k_{D(F)}(h)=k(h(D(F))) \geq 2 \operatorname{vol}\left(\mathbf{R} P^{n}\right)=1$. (Recall that we are using a normalized volume so that $\operatorname{vol}\left(\mathbf{S}^{n}\right)=1$.)

On the other hand, if the developing map $D: \widetilde{M} \rightarrow S^{n}$ is injective so that the holonomy representation is injective, then we have more precise information about the Euler characteristic as follows.

Theorem B. Let $M^{n}$ be an even-dimensional closed projectively flat manifold with amenable fundamental group.

(a) If $\pi_{1} M$ is finite, then $M$ is isomorphic to either $\mathbf{S}^{n}$ or $\mathbf{R} P^{n}$.

(b) If $\pi_{1} M$ is infinite and the developing map is injective, then $\chi(M)=0$.

Proof. If $\pi_{1} M$ is finite, the universal covering $\widetilde{M}$ is compact, and hence the developing map $D: \widetilde{M} \rightarrow S^{n}$, being a local isomorphism, becomes a covering and thus an isomorphism. Now $M$ is isomorphic to $S^{n} / H$, where the holonomy group $H \cong \pi_{1} M$ is finite, and hence is trivial or $\mathbf{Z} / 2$ by considering $\chi(M)$. If a projective transformation $\sigma$ is a free involution on the even-dimensional 
sphere, it is easy to show that $\sigma$ is the antipodal map by looking at the eigenvalues of a linear transformation inducing $\sigma$. This proves (a). If $\pi_{1} M$ is infinite and $D: \widetilde{M} \rightarrow S^{n}$ is injective, we have infinite disjoint developing images of a simplex $\sigma^{n} \in K$. For any finite subset $A \subset H \cong \pi_{1} M$, consider a function $\sum_{h \in A} h \cdot k_{s^{n}}: H \rightarrow \mathbf{R}$, where $s^{n}$ is a fixed developing image of $\sigma^{n}$. (We retain the same notation as in the proof of Theorem A.) Now note that for $g \in H$,

$$
\begin{aligned}
\sum_{h \in A} h \cdot k_{s^{n}}(g) & =\sum_{h \in A} k_{s^{n}}(g h)=\sum_{h \in A} k\left(g h\left(s^{n}\right)\right) \\
& =\sum_{h \in A} 2 \operatorname{vol}\left(g h\left(s^{n}\right)\right) \leq 2 \operatorname{vol}\left(\mathbf{S}^{n}\right)=2 .
\end{aligned}
$$

Hence $2 \geq m\left(\sum_{h \in A} h \cdot k_{s^{n}}\right)=\sum_{h \in A} m\left(h \cdot k_{s^{n}}\right)=\sum_{h \in A} m\left(k_{s^{n}}\right)=|A| m\left(k_{s^{n}}\right)$ and $m\left(k_{s^{n}}\right) \leq 2 /|A|$. Since the cardinality $|A|$ of $A$ may be arbitrarily large, $k\left(\sigma^{n}\right)=m\left(k_{s^{n}}\right)=0$, and hence $\chi(M)=k(M)=\sum_{\sigma^{n} \in K} k\left(\sigma^{n}\right)=0$. Q.E.D.

\section{REFERENCES}

[Go] W. M. Goldman, Geometric structures on manifolds and varieties of representations, Geometry of Group Representations (W. M. Goldman and A. R. Magid, eds.), Contemp. Math., vol. 74, Amer. Math. Soc., Providence, RI, 1988, pp. 169-198.

[Gr] F. P. Greenleaf, Invariant means on topological groups, Van Nostrand Math. Studies, no. 16, Van Nostrand, Princeton, NJ, 1969.

[Ho] H. Hopf, Differential geometry in the large, Lecture Notes in Math., vol. 1000, Springer, Berlin, 1983.

[HT] M. W. Hirsch and W. P. Thurston, Foliated bundles, invariant measures and flat manifolds, Ann. of Math. (2) 101 (1975), 369-390.

[KL] H. Kim and $\mathrm{H}$. Lee, The Euler characteristic of a certain class of projectively flat manifolds, Topology Appl. 40 (1991), 195-201.

[Mi] J. Milnor, On the existence of a connection with curvature zero, Comment. Math. Helv. 32 (1957), 215-223.

[NY] T. Nagano and K. Yagi, The affine structures on the real two-torus, Osaka J. Math. 11 (1974), 181-210.

[Th] W. P. Thurston, The geometry and topology of 3-manifolds, preprint, 1977.

Department of Mathematics, Seoul National University, Seoul 151-742, Korea

E-mail address, H. Kim: HyukKim@KRSNUCC1.BITNET 